\title{
MRI in evaluation of perianal fistulae
}

\author{
Amela Sofic, Serif Beslic, Nedzad Sehovic, Jasmin Caluk, Damir Sofic \\ Institute of Radiology, Clinical Centre of University of Sarajevo, Sarajevo, Bosnia and Herzegovina
}

Received 17 May 2010

Accepted 9 July 2010

Correspondence to: Amela Sofić, MD, MSc, Institute of Radiology, Clinical Centre of University of Sarajevo, Bolnička 25, 71000 Sarajevo, Bosnia and Hercegovina. Phone/Fax:+387 33444 553; E-mail: amelasofic@yahoo.com

Disclosure: No potential conflicts of interest were disclosed.

Background. Fistula is considered to be any abnormal passage which connects two epithelial surfaces. Parks' fistulae classification demonstrates the biggest practical significance and divides fistulae into: intersphincteric, transsphincteric, suprasphincteric and extrasphincteric. Etiology of perianal fistulae is most commonly linked with the inflammation of anal glands in Crohn's disease, tuberculosis, pelvic infections, pelvic malignant tumours, and with the radiotherapy. Diagnostic method options are: RTG fistulography, CT fistulography and magnetic resonance imaging (MRI) of pelvic organs.

Patients and methods. We have included 24 patients with perirectal fistulae in the prospective study. X-rays fistulography, CT fistulography, and then MRI of the pelvic cavity have been performed on all patients. Accuracy of each procedure in regards to the patients and the etiologic cause have been statistically determined.

Results. $29.16 \%$ of transphincteric fistulae have been found, followed by $25 \%$ of intersphincteric, $25 \%$ of recto-vaginal, $12.5 \%$ of extrasphincteric, and $8.33 \%$ of suprasphincteric. Abscess collections have been found in $16.6 \%$ patients. The most frequent etiologic cause of perianal fistulae was Crohn's disease in $37.5 \%$, where the accuracy of classification of MRI was $100 \%$, CT was $11 \%$ and X-rays $0 \%$. Ulcerous colitis was the second cause, with $20.9 \%$ where the accuracy of MRI was $100 \%$, while CT was $80 \%$ and X-rays was $0 \%$. All other etiologic causes of fistulae were found in $41.6 \%$ patients. Conclusions. MRI is a reliable diagnostic modality in the classification of perirectal fistulae and can be an excellent diagnostic guide for successful surgical interventions with the aim to reduce the number of recurrences. Its advantage is that fistulae and abscess are visible without the need to apply any contrast medium.

Key words: perianal fistulae; X-rays; CT; MRI; fistulography; abscess

\section{Introduction}

As per definition, a fistula is any abnormal passage connecting two epithelial surfaces. Anal fistulae have been known ever since the times of Hypocrates and have been described through centuries. In 1835, Frederick Salmon performed a successful operation in London on the writer Charles Dickens. Goodsall describes the fistulous passage in details, and Parks' classification shows the most practical significance until nowadays. The classification refers to classifying fistulae on: intersphincteric, transsphincteric, suprasphincteric and extrasphincteric. In the more detail anatomical classification, the position of fistulae is used ("clockwise") in respect to the clock hands to avoid any misinterpretation. Complex anal fistulae are those which are followed by risk factors: affected external sphincter anal muscle, forward location in women, multiple passages, incontinence, recurrences of fistulae, local radiation, chronic diarrhoea, Crohn's disease. ${ }^{1}$

Aetiology of perianal fistulae is most commonly associated with the inflammation of anal glands in the Crohn's disease, tuberculosis, pelvic infections, pelvic malignant tumours and with radiotherapy. Idiopathic fistulae are rare and are generally explained by chronic intramuscular anal infection (cryptoglandular hypothesis). In about $70 \%$ cases fistulous system drains through skin. Males are affected twice more than females, in ratio $2: 1 .^{2}$

\section{Scope of the study}

The scope of the study is to indicate a possibility of pre-operative diagnostics with magnetic resonance 
TABLE 1. Fistulae classification and the etiology

\begin{tabular}{|c|c|c|c|c|c|c|c|c|}
\hline Patient & age & sex & etiology & Type of fistulae & RTG & Ст & MRI & Absces \\
\hline 1 & 34 & M & Crohn's disease & Intersphincteric & & & + & \\
\hline 2 & 25 & M & Crohn's disease & Transsphincteric & & & + & \\
\hline 4 & 56 & $\mathrm{~F}$ & Ca cerv-radiation & Transsphincteric & & & + & \\
\hline 5 & 33 & M & Crohn's disease & Transsphincteric & & & + & \\
\hline 7 & 28 & M & Crohn's disease & Transsphincteric & & & + & \\
\hline 8 & 33 & M & Crohn's disease & Transsphincteric & & & + & \\
\hline 9 & 25 & $\mathrm{~F}$ & Unknown & Intersphincteric & & & + & \\
\hline 10 & 44 & M & Unknown & Suprassphincteric & & + & + & + \\
\hline 11 & 56 & $\mathrm{~F}$ & Crohn's disease & Suprassphincteric & & + & + & + \\
\hline 15 & 19 & M & Ulcerous colitis & Extrasphincteric & + & + & + & \\
\hline 16 & 55 & $\mathrm{~F}$ & Ca recti-radiation & Rectovaginal & + & + & + & \\
\hline 17 & 45 & $\mathrm{~F}$ & Infla.dermoid cysts & Rectovaginal & + & + & + & + \\
\hline 18 & 30 & $\mathrm{~F}$ & Postpartum & Rectovaginal & + & + & & \\
\hline 19 & 34 & $\mathrm{~F}$ & Ulcerous colitis & Extrasphincteric & + & + & + & + \\
\hline 20 & 44 & $\mathrm{~F}$ & Ulcercous colitis & Transsphincteric & & + & + & \\
\hline 21 & 42 & M & Ulcerous colitis & Extrasphincteric & + & + & + & \\
\hline 22 & 54 & M & Ca recti & Intersphincteric & & & + & \\
\hline
\end{tabular}

imaging (MRI) in viewing and classifying perianal fistulae, with the aim to have as successful surgical treatment as possible, without recurrences. From the practical surgical point of view, it is of crucial importance to avoid incontinence as a post-operative complication.

\section{Patients and methods}

From the 2008 to 2009 we included in the prospective study 24 patients with existing perirectal fistulae. The average age of patients was $41.45 \pm 2.6$ years, ranging from 19 to 67 years, with the same number of females $50 \%(n=12)$ and males $50 \%(n=12)$.

X-rays fistulography was performed first on all patients, followed by CT fistulography, and then by MRI of the pelvis. X-rays fistulography was performed on X-ray diascope (Practix 100, Philips, Aidhoven, the Netherlands), and after the application of Ultravist contrast medium through perianal fistulous openings. Immediately after that procedure, while the fistulous system was filled with the same contrast medium, the pelvic CT was performed on MDCT (Volume zoom, Siemens, Erlangen, Germany). The MRI pelvic examination was performed on $1.5 \mathrm{~T}$ machine (Avanto, Siemens, Erlagen, Germany) by using a routine protocol for pelvic examinations containing T1 3-plane views (axial, coronal and saggital planes), T2 axial and saggital planes, as well as bi-plane (axial and saggital planes) without the application of a contrast medium.

Based on the data obtained, a comparison of all findings has been done, using each particular method. 

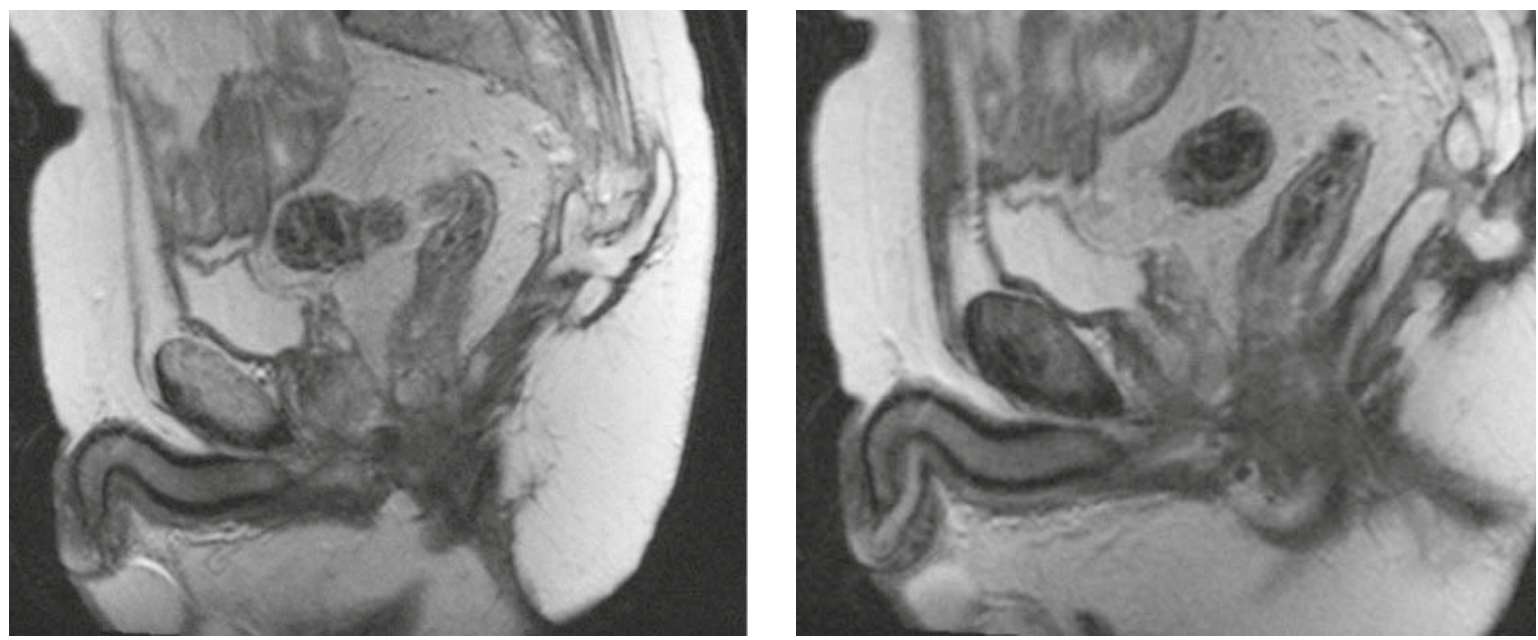

FIGURE 1. Suprasphincteric fistula with the abscess collection in the gluteal region (T2W, sagital).
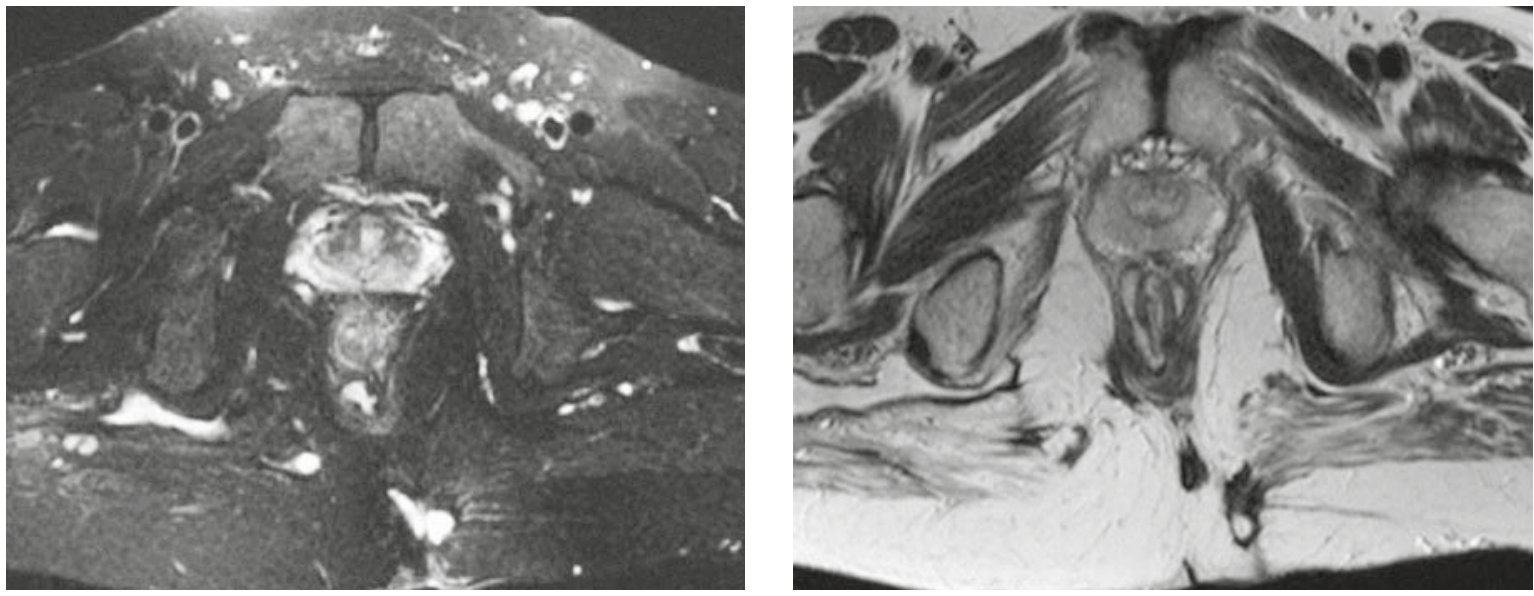

FIGURE 2. Intersphincteric fistula in the shape of a horseshoe with the opening to the left posterior ( $\mathrm{T} 2 \mathrm{Fs}$ and T2W tra).

\section{Results}

The following has been classified in 24 patients: transsphincteric fistulae in $29.16 \%$ of patients $(\mathrm{n}=$ $7)$, intersphincteric in $25 \%(n=6)$, rectovaginal in $25 \%(n=6)$, extrasphincteric in $12.5 \%(n=3)$ and suprasphincteric in $8.33 \%$ of patients $(n=2)$. Abscess collections were found in $16.6 \%$ of patients $(n=4)$ (Table 1).

Only 9 fistulae were identified by X-rays fistulography; 12 fistulae were identified by CT fistulography; and 20 by MRI (Table 1). For identifying fistulous ration in respect to the sphincter complex, MRI was a superior method, while for rectovaginal fistulae, $\mathrm{CT}$ and X-rays fistulography were better.

The most frequent cause of fistulae in our sample was Crohn's disease with $37.5 \%(n=9)$, followed by ulcerous collitis with $20.9 \%(n=5)$, rectal cancer $16.7 \%(\mathrm{n}=4)$, postpartum $8.3 \%(\mathrm{n}=2)$, unknown aetiology $8.3 \%(n=2)$, cervical cancer $4.2 \%$ $(\mathrm{n}=1)$, and inflammatory dermoid cysts $4.2 \%$ $(\mathrm{n}=1)$ (Table 1).

$X$-rays fistulography demonstrated the accuracy of $37.5 \%$. $\left(\chi^{2}=4.444, \mathrm{p}=0.035\right)$, CT fistulography had accuracy of $50 \%,(\chi 2=6.000, p=0.014)$, and the MRI demonstrated accuracy of $83.3 \%$. $\left(\chi^{2}=4.800, \mathrm{p}=0.028\right)$ (Table 2).

The comparison of results in respect to the sex of the patients demonstrated that there were differences in accuracy in favour of female patients (Table 2). Twenty-six \% of all fistulae in our study are rectovaginal, where the total accuracy in females is on increase. The accuracy of X-rays fistulography in females was $75 \%$ vs. males $25 \%$. The MRI accuracy for females was $66 \%$ vs. males 33\%. Slightly higher accuracy for females was demonstrated in CT fistulography, in respect to the MRI, 
TABLE 2. Accuracy of all three procedures

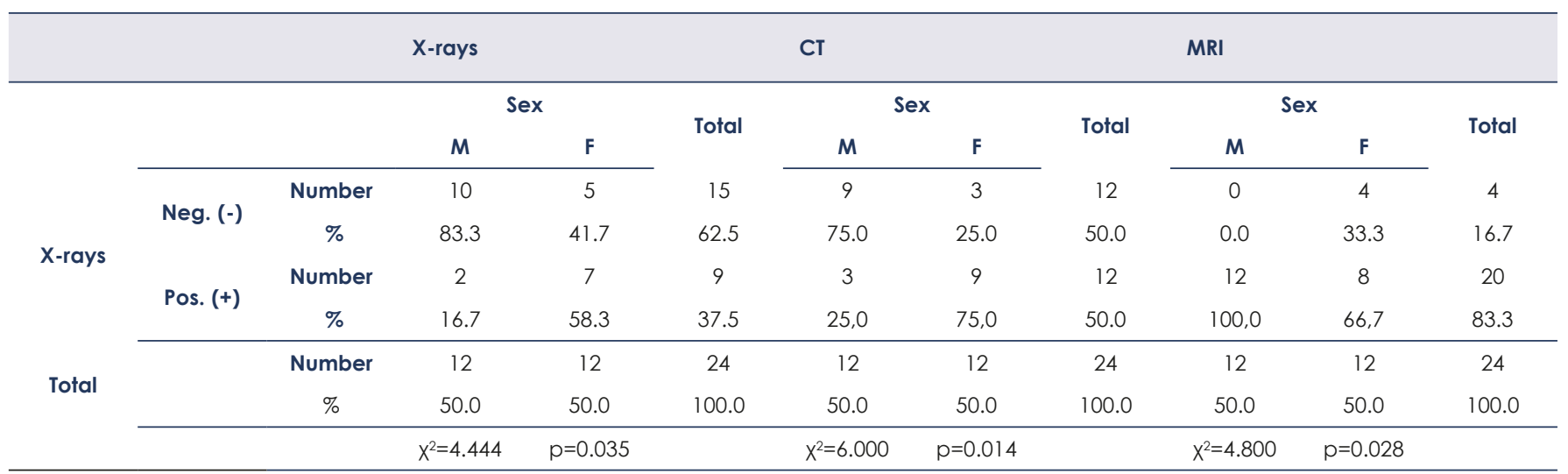

TABLE 3. The accuracy of procedures varies depending on the aetiology - X-rays

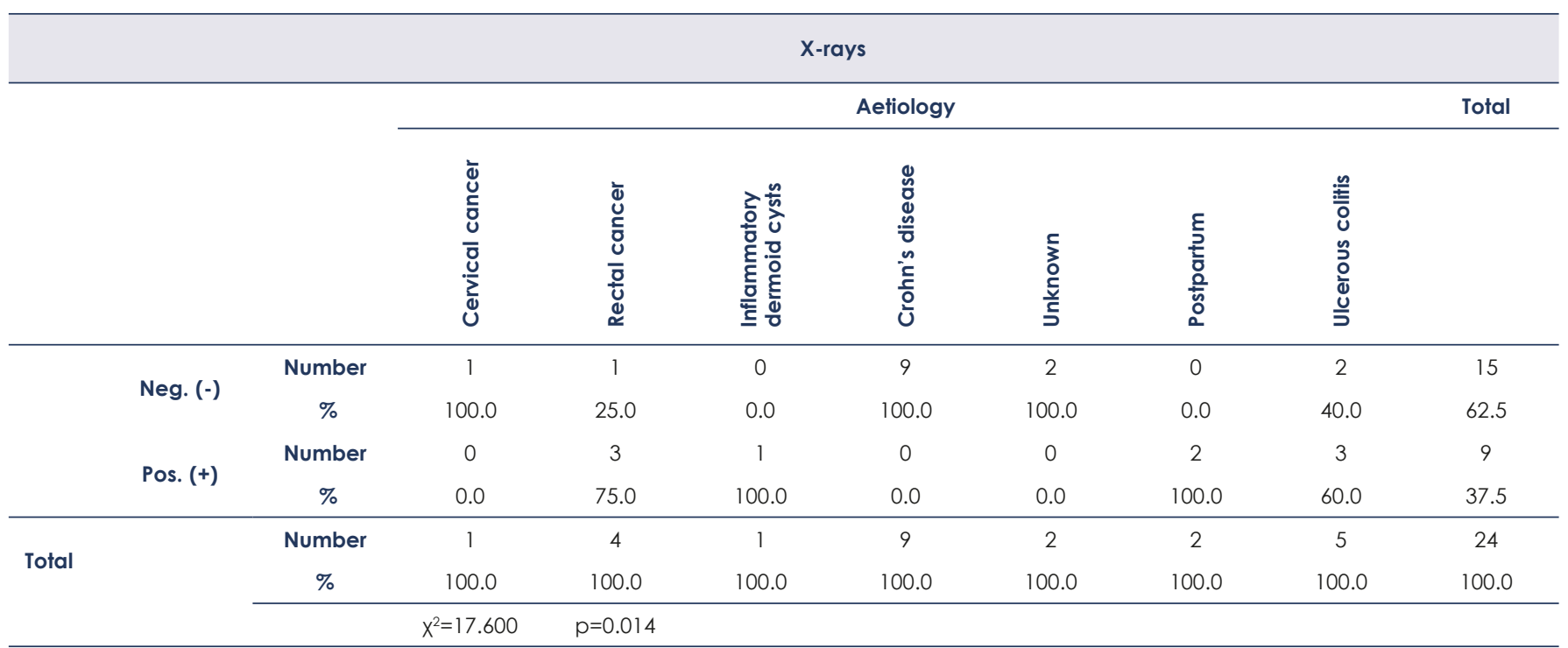

because it identified more rectovaginal fistulae compared to the MRI. The lowest accuracy was found in X-rays fistulography.

The accuracy of procedures varies depending on the aetiology as well. X-rays fistulography demonstrates the accuracy in identifying: ulcerous colitis $60 \%$, Crohn's disease $0 \%$, rectal cancer $75 \%$, cervical cancer $0 \%$, inflammatory dermoid cysts $100 \%$, postpartum $100 \%$ and of unknown aetiology $0 \%$ (Table 3.). CT fistulography demonstrates the accuracy in identifying: ulcerous colitis $80 \%$, Crohn's disease $11.1 \%$, rectal cancer $75 \%$, cervical cancer $0 \%$, inflammatory dermoid cyst $100 \%$, postpartum $100 \%$, and of unknown aetiology $50 \%$ (Table 4.). MRI demonstrates the accuracy in identifying: ulcerous colitis $100 \%$, Crohn's disease $100 \%$, rectal cancer $50 \%$, cervical cancer $100 \%$, in- flammatory dermoid cyst $100 \%$, postpartum $0 \%$, and of unknown aetiology $100 \%$ (Table 5.) (Figures $1,2,3)$.

\section{Discussion}

Not so long time ago, surgeons performed operations on perirectal fistulae without the previous radiological assessment. The surgical examination under anaesthetics (EUA) consisted of visual inspection, palpation with the probe of a fistulous passage under general anaesthesia. Numerous diagnostic modalities failed in visualisation and classification of perianal fistulae.

Fistulography, as the earliest X-rays method, cannot classify fistulae due to the inadequate 

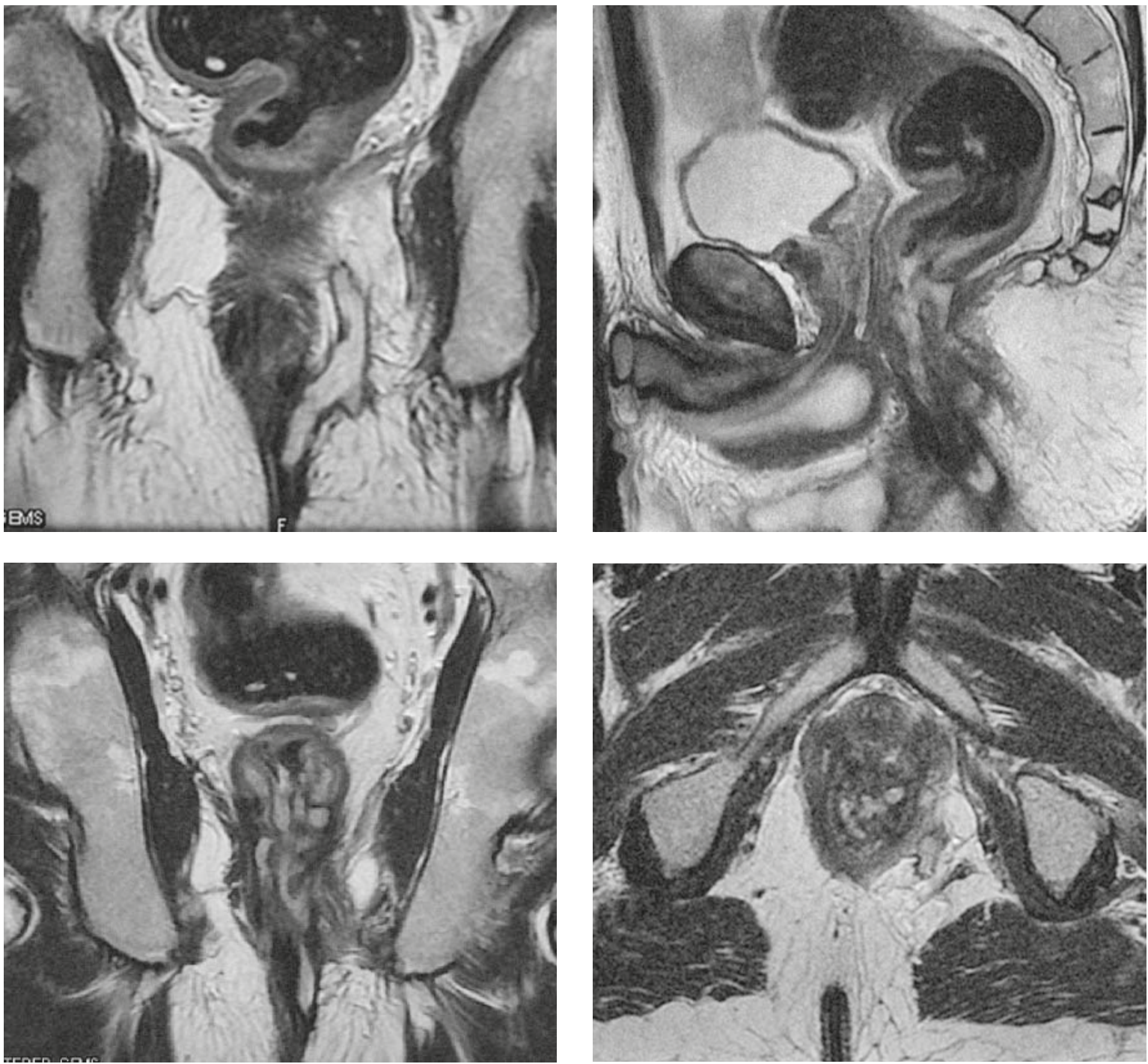

FIGURE 3. A complex transsphincteric fistula to the left (T2W cor,T2W sag,T2W tra).

showing of anatomic structures, so that frequently it is unclear and difficult for the interpretation. CT can identify the existence of fistulous passages, either through non-ionic water solluble contrast media being inserted per rectum or through the fistulous opening. However, it is not sufficiend for a more detailed analysis of the whole complex of primary and numerous secondary branches in the fistulous system. Although the application of multidetector CT fistulography with the option of isotropic voxels and multiplaned imaging can bridge the aforementioned issues, researchers do not show enough interest in this field. It is obvious that the superiority of MRI and endorectal ultrasound (EUS) examination in the evaluation of perirectal fistulae provides a better motivation to the researchers. ${ }^{3}$
MRI with the superficial body-coil, besides other anatomic structures in the pelvis, shows excellent results in showing rectum, perianal region, internal, external anal sphincter, levator ani, ishiorectal and ishioanal region. ${ }^{4}$ In the evaluation of perirectal fistulae, it is very important to describe the relationship between fistula and the sphincteric mechanism in the coronal plane (Figure 3). It is equally important to describe the primary fistulous passage as well as the secondary ramification and possible associated abscess due to axial images. Images in the saggital plane are suitable for showing rectovaginal fistulae and the pre-sacral region. The surgical exploration without previous MRI diagnostics can be made difficult due to the presence of fibrosis and oedema, so that the MRI can identify the hidden intersphincteric space with the cap- 
TABLE 4. The accuracy of procedures varies depending on the aetiology - CT

\begin{tabular}{|c|c|c|c|c|c|c|c|c|c|c|}
\hline & & & \multicolumn{7}{|c|}{ Aetiology } & \multirow[t]{2}{*}{ Total } \\
\hline & & & 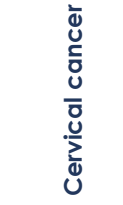 & 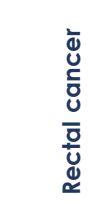 & 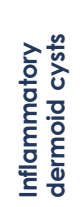 & 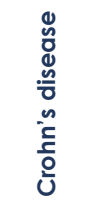 & 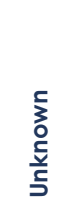 & 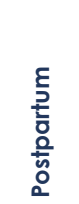 & 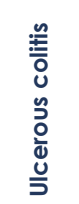 & \\
\hline & \multirow{2}{*}{ Neg. (-) } & Number & 1 & 1 & 0 & 8 & 1 & 0 & 1 & 12 \\
\hline & & $\%$ & 100.0 & 25.0 & 0.0 & 88.9 & 50.0 & 0.0 & 20.0 & 50.0 \\
\hline & \multirow{2}{*}{ Pos. (+) } & Number & 0 & 3 & 1 & 1 & 1 & 2 & 4 & 12 \\
\hline & & $\%$ & 0.0 & 75.0 & 100.0 & 11.1 & 50.0 & 100.0 & 80.0 & 50.0 \\
\hline
\end{tabular}

TABLE 5. The accuracy of procedures varies depending on the aetiology - MRI

\begin{tabular}{|c|c|c|c|c|c|c|c|c|c|c|}
\hline & & & \multicolumn{7}{|c|}{ Aetiology } & \multirow[t]{2}{*}{ Total } \\
\hline & & & 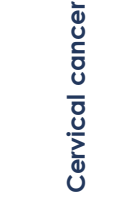 & 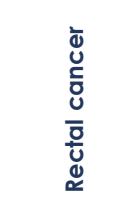 & 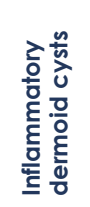 & 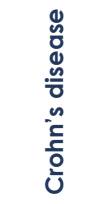 & $\begin{array}{l}\frac{5}{3} \\
0 \\
\frac{5}{5} \\
5\end{array}$ & $\begin{array}{l}\varepsilon \\
\frac{5}{1} \\
\text { 읗 } \\
\text { o. }\end{array}$ & 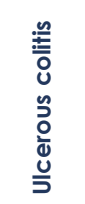 & \\
\hline & \multirow{2}{*}{ Neg. (-) } & Number & 0 & 2 & 0 & 0 & 0 & 2 & 0 & 4 \\
\hline & & $\%$ & 0.0 & 50.0 & 0.0 & 0.0 & 0.0 & 100.0 & 0.0 & 16.7 \\
\hline & \multirow{2}{*}{ Pos. (+) } & Number & 1 & 2 & 1 & 9 & 2 & 0 & 5 & 20 \\
\hline & & $\%$ & 100.0 & 50.0 & 100.0 & 100.0 & 100.0 & 0.0 & 100.0 & 83.3 \\
\hline
\end{tabular}

tured puss without rupturing the cutaneous layer. It is also useful in high fistulae - both transsphincteric and extrasphincteric. Fistulous passage, abscess, as well as all inflamed structures, show on T2W sequences high signal intensity, so they are easily noticed in respect to the muscles which have low signal intensity. On non-contrasted T1W sequences, fistulous passages (especially the secondary ones, the smaller ones), as well as abscess collections are identified with difficulties due to the moderate signal intensity of normal structures of the sphincter muscle and levatore ani. Therefore, it is recommended to use sequences with suppressed fats as the i.v. application of Gadolinium as a contrast medium. The fistulous opening on skin can be filled with Gadolinium, thus obtaining the picture of fistulous system in hipersignal in T1W time, or by a cheaper method - by injecting physiological solution with showing the fistulous system in hipersignal in T2W time.

There is not a large number of published studies in relation to the fistulography. Thus, Kuipers and Schulpen as far as in 1985 emphasized that fistulography is a reliable and accurate method in 
only $16 \%$ cases. ${ }^{5}$ The best results were obtained by Weisman et al. who managed to obtain reliable fistulography in half of the examined cases - 27 patients in his study, which generally represents only very modest results. ${ }^{6}$

The first reporting on the accuracy of MRI in detection and classification of perianal fistulae was from 1992 - 1994 in Lunnis' publications, with the matching of $86-88 \%$ between MRI and surgical findings. ${ }^{7,8}$ EUS is not a comfortable procedure, however, it has a very good spatial resolution due to the close contact of the probe $(10 \mathrm{MHZ})$ with the rectal wall, which gives it the superiority in evaluation of fistulous openings within the rectum and the level of disruption of the sphincter in post-surgically incontinent patients. MRI is more comfortable $^{4}$ and better shows intersphincteric abscesses. Halligan and Stoker claim that MRI assists the surgeon to reduce post-surgical recurrences for $75 \%$, and that the EUS is only an alternative when it is not possible to perform the MRI. ${ }^{9}$ Hussain et al. from the Netherlands, in his study from 1996, talked about the superiority of MRI in classification of fistulae compared to the EUS (89 vs 61\%). ${ }^{10}$ Spenser's study from 1996, in which body-coil was used, demonstrated the accuracy of the MRI in showing perirectal fistulae of $88 \%{ }^{11}$ The same author, two years later, in 1988, obtained the classification of fistulae by EUS of $81 \%$, and by the MRI of $90 \% .^{12}$ Sensitivity of the primary fistulous passage reaches up to $100 \%$, however, with the specificity of $86 \%$. Yee et al. concludes in his study that the native endoscopic ultrasound does not detect rectovaginal fistulae. ${ }^{13}$

In the recent times, ultrasound visibility of fistulae filled with peroxide has been researched, which is strictly recommended for the pre-surgical evaluation in patients with recurrences of fistulae. Gustafson et al. claimed that the endoscopic EUS complemented the MRI in classification of fistulae. ${ }^{14}$

The optimal diagnostic approach is a combination of EUS, examination under anaesthesia (EUA) and MRI in patients with fistulae who have the Crohn's disease, reports Schwartz et al. in his article. ${ }^{15}$ The Viennese group of radiologists, in their study, obtains the accuracy in classification of fistulae by the MRI of $84 \%$, vs EUS of $60 \%{ }^{16}$

Gravante and Giordano in 2008 promoted 3D EUS as equal to the MRI in the examination of fistulae. The accuracy of the MRI was obtained in the range of $90 \%$ compared with the EUS which obtained $81 \% .{ }^{17}$ Schratter-Sehn et al. obtained higher sensitivity by the EUS of $82 \%$ compared to n CT of $24 \%$ in the classification of perianal fistulae in the Crohn's disease, while Schaefer et al. obtains the matching with the surgical findings of $89 \% .18,19$ In our study, we obtained the accuracy in classification of fistulae by X-rays of $37.5 \%$, with the CT accuracy was $50 \%$, and the MRI accuracy was $83 \%$. The most frequent aetiological cause of perianal fistulae was Crohn's disease of $37.5 \%$, where the accuracy of classification by the MRI was $100 \%$, by CT of $11 \%$ and by X-rays was $0 \%$. Ulcerous colitis was on the second place with $20.9 \%$, where the accuracy in that respect was $100 \%$ by the MRI, $80 \%$ by $\mathrm{CT}$ and $0 \%$ by X-rays. All other etiological causes of fistulae including radiation, which is a serious side effect after oncological treatment ${ }^{20,21}$, were $41.6 \%$. Abscess collection was found in 16.6 patients.

\section{Conclusions}

MRI is a reliable diagnostic modality in the classification of perirectal fistulae and can be an excellent diagnostic guide for successful surgical interventions with the aim to reduce the number of recurrences. Its advantage is that fistulae and abscess are visible without the need to apply any contrast medium.

\section{References}

1. Whiteford MH. Perianal abscess/fistula disease. Clin Colon Rectal Surg 2007; 20: 102-9.

2. Sainio P. Fistula-in-ano in a defined population. Incidence and epidemiological aspects. Ann Chir Gynaecol 1984; 73: 219-24.

3. Jones J, Tremaine W. Evaluation of perianal fistulas in patients with Crohn's disease. Med Gen Med 2005; 7: 16.

4. Sofić A, Šehović N, Bešlić Š, Prnjavorac B, Bilalović N, Čaluk J, et al. MR rectum imaging with ultra sound gel as instrumental contrast media in tubulovillous adenoma. Radiol Oncol 2008; 42: 136-42.

5. Kuijpers HC, Schulpen T. Fistulography for fistula-in-ano. Is it useful? Dis Colon Rectum 1985; 28: 103-4.

6. Weisman RI, Orsay CP, Pearl RK, Abcarian H. The role of fistulography in fistula-in-ano. Report of five cases. Dis Colon Rectum 1991; 34: 181-4.

7. Lunniss PJ, Armstrong P, Barker PG, Reznek RH, Phillips RK. Magnetic resonance imaging of anal fistulae. Lancet 1992; 340: 394-6.

8. Lunniss PJ, Barker PG, Sultan AH, Armstrong P, Reznek RH, Bartram Cl, et al. Magnetic resonance imaging of fistula-in-ano. Dis Colon Rectum 1994; 37: 708-18.

9. Halligan S, StokerJ. Imaging of fistula in ano. Radiology 2006; 239: 18-33.

10. Hussain SM, Stoker J, Schouten WR, Hop WC, Lameris JS. Fistula in ano: endoanal sonography versus endoanal MR imaging in classification. Radiology 1996; 200: 475-81.

11. Spencer JA, Ward J, Beckingham IJ, Adams C, Ambrose NS. Dynamic contrast enhanced MR imaging of perianal fistulas. AJR Am J Roentgenol 1996; 167: 735-41.

12. Spencer JA, Chapple K, Wilson D, Ward J, Windsor AC, Ambrose NS. Outcome after surgery for perianal fistula: Predictive value of MR imaging. AJR Am J Roentgenol 1998; 171: 403-6. 
13. Yee LF, Birnbaum EH, Read TE, Kodner IJ, Fleshman JW. Use of endoanal ultrasound in patients with rectovaginal fistulas. Dis Colon Rectum 1999; 42: 1057-64.

14. Gustafsson UM, Kahvecioglu B, Astrom G, Ahlstrom H, Graf W. Endoanal ultrasound or magnetic resonance imaging for preoperative assessment of anal fistula: a comparative study. Colorectal Dis 2001; 3: 189-97.

15. Schwartz DA, Wiersema MJ, Dudiak KM, Fletcher JG, Clain JE, Tremaine WJ, et al. A comparison of endoscopic ultrasound, magnetic resonance imaging, and exam under anesthesia for evaluation of Crohn's perianal fistulas. Gastroenterology 2001; 121: 1064-72.

16. Maier AG, Funovics MA, Kreuzer SH, Herbst F, Wunderlich M, Teleky BK, et al. Evaluation of perianal sepsis: comparison of anal endosonography and magnetic resonance imaging. J Magn Reson Imaging 2001; 14: 254-260.

17. Gravante G, Giordano P. The role of three-dimensional endoluminal ultrasound imaging in the evaluation of anorectal diseases: a review. Surg Endosc 2008; 22: 1570-8.

18. Schratter-Sehn AU, Lochs $\mathrm{H}$, Vogelsang $\mathrm{H}$, Schurawitzki $\mathrm{H}$, Herold $\mathrm{C}$ Schratter M. Endoscopic ultrasonography versus computed tomography in the differential diagnosis of perianorectal complications in Crohn's disease. Endoscopy 1993; 25: 582-6.

19. Schaefer $O$, Lohrmann $C$, Langer $M$. Assessment of anal fistulas with highresolution subtraction MR-fistulography: comparison with surgical findings. J Magn Reson Imaging 2004; 19: 91-8.

20. Botros M, Quevedo JF, Miller RC. Angiosarcoma of the liver after multimodality therapy for gallbladder carcinoma. Radiol Oncol 2009; 43: 126-31.

21. Piekarski JH, Jereczek-Fossa BA, Nejc D, Pluta P, Szymczak W, Sek P, et al. Does fecal diversion offer any chance for spontaneous closure of the radiation-induced rectovaginal fistula? Int J Gynecol Cancer 2008; 18: 66-70. 